\title{
Estimation of End-Diastolic Pressure via Deconvolution
}

\author{
Christoph Hoog Antink, Daniel Rüschen, Steffen Leonhardt, Marian Walter \\ Philips Chair for Medical Information Technology, RWTH Aachen University, Germany
}

\begin{abstract}
Left ventricular assist devices (LVADs) can significantly improve survival rate and quality of life for patients suffering from end-stage heart failure. Several promising strategies to control LVADs are being developed, some being focused on the end-diastolic pressure (EDP). For those, the problem of EDP estimation in real-time has to be solved. In this work, a deconvolution-based method to identify features in cardiac signals is presented. This method is applied to the estimation of the EDP from the left-ventricular pressure (LVP) signal and evaluated on animal trial data. In 11 trials with adult sheep, a myocardial infarction was induced and an LVAD was implanted. A total of 37.6 hours of LVP data was annotated by a medical expert. Compared to the annotations, a root mean square error of $11.6 \mathrm{~ms} /$ $4.1 \mathrm{mmHg}$ was achieved using the proposed deconvolution method.
\end{abstract}

\section{Introduction}

According to the American Heart Association, about 5.7 million Americans live with heart failure. For patients suffering from its end-stage manifestation, left ventricular assist devices (LVADs) can significantly improve survival rate and quality of life [1]. For the control of LVADs, several strategies exist [2], one promising approach being the control of the end diastolic pressure (EDP). EDP is defined as the end-diastolic (ED) value of the left ventricular pressure (LVP). Thus, to implement an EDP control strategy, its value has to be determined in real time.

In [3], the estimation of the ED time point was approach by an analysis of the peak curvature of the LVP signal and evaluated in terms of temporal accuracy. Here, a deconvolution-based approach is presented and its accuracy is evaluated both in temporal as well as amplitudinal accuracy. In previous work we have demonstrated that deconvolution methods have a great potential in the processing of (multimodal) cardiac signals. It was shown in [4] that blind deconvolution can be used to estimate a virtual source signal and linear filter coefficients to analyze and represent measured multimodal signals (e.g. photoplethysmography and ballistocardiography). In [5] it was further shown that a desired, measured signal (e.g. reference ECG) can be approximated by filtering measured multichannel signals (e.g. capacitively coupled ECG) with estimated linear filters. In this work, we expand the concept of blind deconvolution towards the estimation of the EDP. In particular, a measured signal and a desired, virtual signal are used to estimated linear filter coefficients in a training phase. These filter coefficients can subsequently be used to efficiently locate ED time points via convolution and peak detection.

\subsection{Deconvolution Algorithm}

The aim of the proposed algorithm is to find a feature, namely the ED time point, in the LVP signal. Thus, the observed signal $\vec{x}=x(t)$, with $t \in 0 \ldots T-1$, is the LVP signal, see Figure 1, top graph, solid line. The desired signal $\vec{y}=y(t)$ is derived from the temporal location of the ED time points. In particular, a medical expert has identified all points in time $\vec{t}_{\mathrm{EDP}}$ that correspond to an EDP, see Figure 1, top graph, crosses. Now, the desired signal $y(t)$ is defined as a train of impulses:

$$
y(t)= \begin{cases}1 & \text { for } t \in \vec{t}_{\mathrm{EDP}} \\ 0 & \text { otherwise }\end{cases}
$$

In the next step, the FIR filter coefficients $\vec{a}=$ $a(0), \ldots, a(q)$ have to be identified that transform $x(t)$ into an estimation of the desired signal,

$$
\tilde{y}(t)=a(t) * x(t)=\sum_{\tau=0}^{q} a(\tau) x(t-\tau),
$$

with the convolution operator $*$. The estimation of $\vec{a}$ can be formulated as a least-squares minimization problem,

$$
\vec{a}=\underset{\vec{a}}{\arg \min }\left|y(t)-\sum_{\tau=0}^{q} a(\tau) x(t-\tau)\right|^{2} .
$$

This problem can be solved efficiently in the Fourier domain, where the convolution is represented by a multiplication. Thus, the equation

$$
\tilde{Y}(\omega)=\sum_{\tau=0}^{q} a(\tau) X(\omega) e^{-j 2 \pi \omega \tau / T}
$$




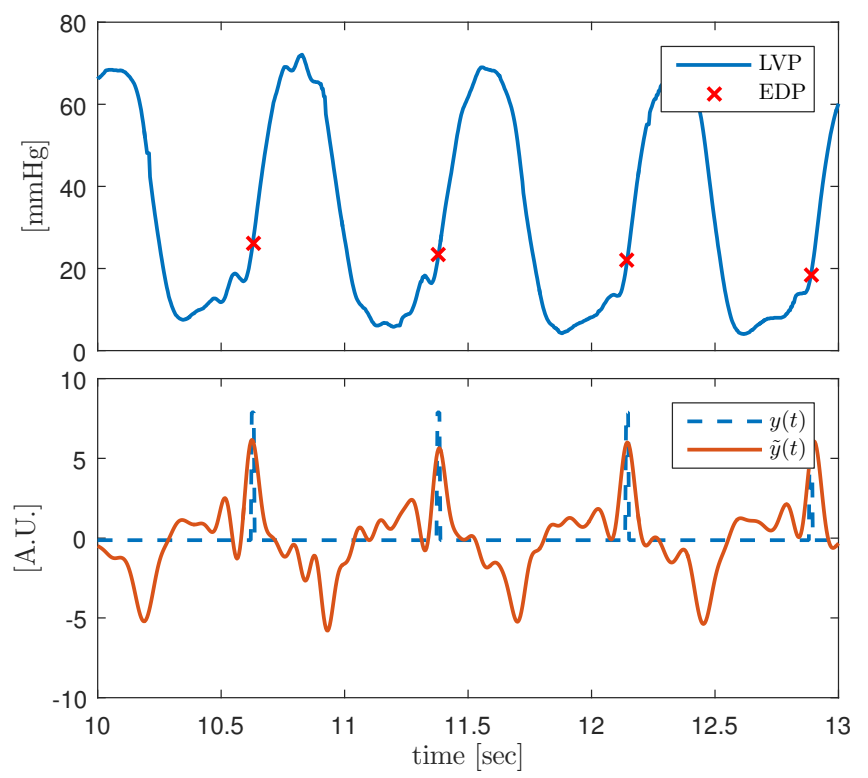

Figure 1. The top graph shows a segment of the LVP signal $\left(x(t)\right.$, solid line) and annotated EDPs ( $\vec{t}_{\mathrm{EDP}}$, crosses) of trial 3. The bottom graph shows the desired virtual signal $(y(t)$, dashed line) and the estimation reconstructed via linear filtering $(\tilde{y}(t)=a(t) * x(t)$, solid line).

can be rewritten as

$$
\tilde{Y}(\omega)=\vec{a} \cdot \vec{E}(\omega) \cdot X(\omega)
$$

with the Fourier-domain delay-vector

$$
\vec{E}(\omega)=E(n, \omega)=\left(1, e^{-j 2 \pi \omega / T}, \ldots, e^{-j 2 \pi \omega q / T}\right)^{T}
$$

with $n \in 0 \ldots q$, see also [6]. Let $(\cdot)^{T}$ be the transpose, $(\cdot)^{H}$ the Hermitian transpose and $(\cdot)^{*}$ the conjugation operator. Now, the $l 2$-optimal vector of filter coefficients $\vec{a}$ can be obtained via

$$
\begin{aligned}
\vec{a}= & \left(\vec{Y}^{*} \cdot \mathbf{M}^{T}+\vec{Y} \cdot \mathbf{M}^{H}\right) \cdot \\
& \left(\mathbf{M}^{*} \cdot \mathbf{M}^{T}+\mathbf{M} \cdot \mathbf{M}^{H}\right)^{-1},
\end{aligned}
$$

with the matrix

$$
\mathbf{M}=(\vec{E}(0) \cdot X(0), \ldots, \vec{E}(T-1) \cdot X(T-1)) .
$$

From the signal $\tilde{y}(t)$, ED time points are determined by peak detection.

\subsection{Experimental Details}

The LVP data used in this study was gathered in 11 animal experiments with adult sheep (Ovis aries, Rhön, female, approx. $63 \mathrm{~kg}$ ) with experimentally induced myocardial infarction. The LVP was obtained using two different sensor systems: A conventional pressure measurement system utilizing a fluid filled catheter between the LV compartment and the sensor unit (Xtrans, CODAN pvb Critical Care GmbH, Forstinning, Germany, type 'A' in the following) and an experimental optical sensor (type 'B', employing the measurement principle introduced in [7]), mounted on the inlet of a rotary blood pump (Impella CP, Abiomed Inc., Danvers, USA).

At the beginning of each experiment, the sheep were put under general anesthesia. Subsequently, a myocardial infarction was triggered by balloon occlusion of the left anterior descending artery followed by an injection of microspheres into the occluded vessels. The Impella CP was implanted directly after the first symptoms of myocardial infarction were noticeable. The study was approved by the corresponding office at the RWTH Aachen Faculty of Medicine in accordance with national and international standards.

To identify the EDP, LVP signals were manually annotated by a medical expert. In the LVP signal, the EDP is visible as a "corner" or "bend". Table 1 provides details about the sensor type, number of EDPs annotated, total time of annotated signal, mean heart rate as well as mean and standard deviation (SD) of the EDP.

\begin{tabular}{ccrrrr}
$\begin{array}{c}\text { trial } \\
\#\end{array}$ & $\begin{array}{c}\text { sens. } \\
\text { type }\end{array}$ & $n_{\text {ann }}$ & $\begin{array}{c}t_{\text {ann }} \\
{[\mathrm{h}]}\end{array}$ & $\begin{array}{c}\overline{H R} \\
{[\mathrm{BPM}]}\end{array}$ & $\begin{array}{c}\overline{E D P} \pm \mathrm{SD} \\
{[\mathrm{mmHg}]}\end{array}$ \\
\hline 1 & $\mathrm{~B}$ & 2388 & 0.8 & 49 & $27.2 \pm 4.2$ \\
2 & $\mathrm{~B}$ & 19151 & 3.9 & 83 & $15.6 \pm 6.4$ \\
3 & $\mathrm{~B}$ & 19726 & 3.8 & 87 & $19.7 \pm 6.5$ \\
4 & $\mathrm{~B}$ & 23166 & 3.6 & 106 & $13.5 \pm 5.4$ \\
5 & $\mathrm{~B}$ & 18535 & 3.1 & 99 & $21.7 \pm 8.8$ \\
6 & $\mathrm{~B}$ & 21615 & 4.6 & 79 & $20.6 \pm 6.5$ \\
7 & $\mathrm{~B}$ & 927 & 0.2 & 84 & $22.1 \pm 3.8$ \\
8 & $\mathrm{~A}$ & 13608 & 2.4 & 94 & $36.8 \pm 9.5$ \\
9 & $\mathrm{~A}$ & 18156 & 3.5 & 87 & $16.0 \pm 9.4$ \\
10 & $\mathrm{~A}$ & 23630 & 5.4 & 73 & $13.3 \pm 6.3$ \\
11 & $\mathrm{~A}$ & 40983 & 6.4 & 107 & $10.9 \pm 10.7$ \\
\hline & $\sum$ & 201885 & 37.6 & $\varnothing 89$ & $27.2 \pm 4.2$
\end{tabular}

Table 1. Details on the 11 animal trials including total number of annotated EDP, total duration of annotated signal as well as gross statistics for HR and EDP.

\subsection{Algorithmic Details}

Data was recorded at $f_{\mathrm{s}}^{\prime}=1 \mathrm{kHz}$ and downsampled to $f_{\mathrm{s}}=250 \mathrm{~Hz}$ for computational reasons. Before deconvolution, a fourth order Butterworth bandpass filter with a passband of 0.1 to $10 \mathrm{~Hz}$ to filter out the DC component and noise was applied. To find the peaks in the estimated desired signal, a basic peak detection algorithm with an adaptive threshold was implemented. Parameters of the peak detector were kept constant for all trials. 
To assess the performance of the algorithm, crossvalidation was performed. Here, the annotated date of one animal trial was used to estimate the FIR filter coefficients $\vec{a}$. Using this filter, the EPD was estimated in the remaining trials and compared to the human annotation. An EDPpoint was considered missed and excluded if a threshold of $t_{\mathrm{th}}=50 \mathrm{~ms}$ was exceeded.

\section{Results and Discussion}

Figure 2 shows the influence of the length $q$ of the filter $\vec{a}$ on the temporal root mean square error (RMSE) as well as the mean and median error. For $q=1$, a large RMSE as

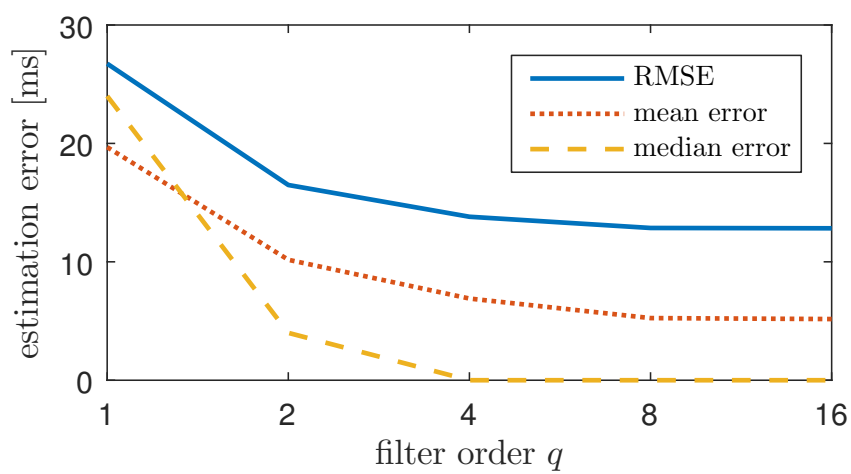

Figure 2. Gross cross-validated RMSE, mean and median error for different filter orders $q$.

well as a systematic offset, indicated by the mean and median error, can be observed. This can be explained by the observation that for $q=1, \vec{a}$ becomes a simple differentiator. Thus, the EDP point is estimated to be at the largest slope of the LVP signal, which is consistent with the findings reported in [3]. For $q>8$, no notable improvement in terms of RMSE and offset can be achieved.

Figure 3 shows the percentage of missed as well as falsely detected EDP time points. It is evident that, using

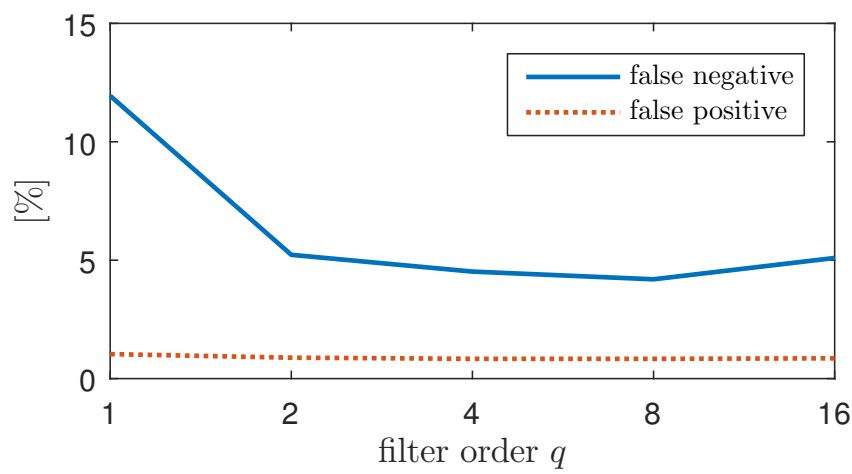

Figure 3. Gross FPR and FNR for different filter orders $q$.

a basic peak detection strategy, a low false positive rate of about $1 \%$ can be achieved. At the same time, the percentage of EDP time points missed ranges between $13 \%$ and $5 \%$ and increases for $q>8$. It should be noted that this increase in false negatives is attributed to shortcomings of peak-selection strategy.

Figure 4 shows the distribution of EPD estimation error per trial for $q=8$. For this analysis, cross-validation was used, i.e., all filters were used to predict the EDP for one case except for the filter trained on this specific dataset. After that, averaging was performed. Numeric details are
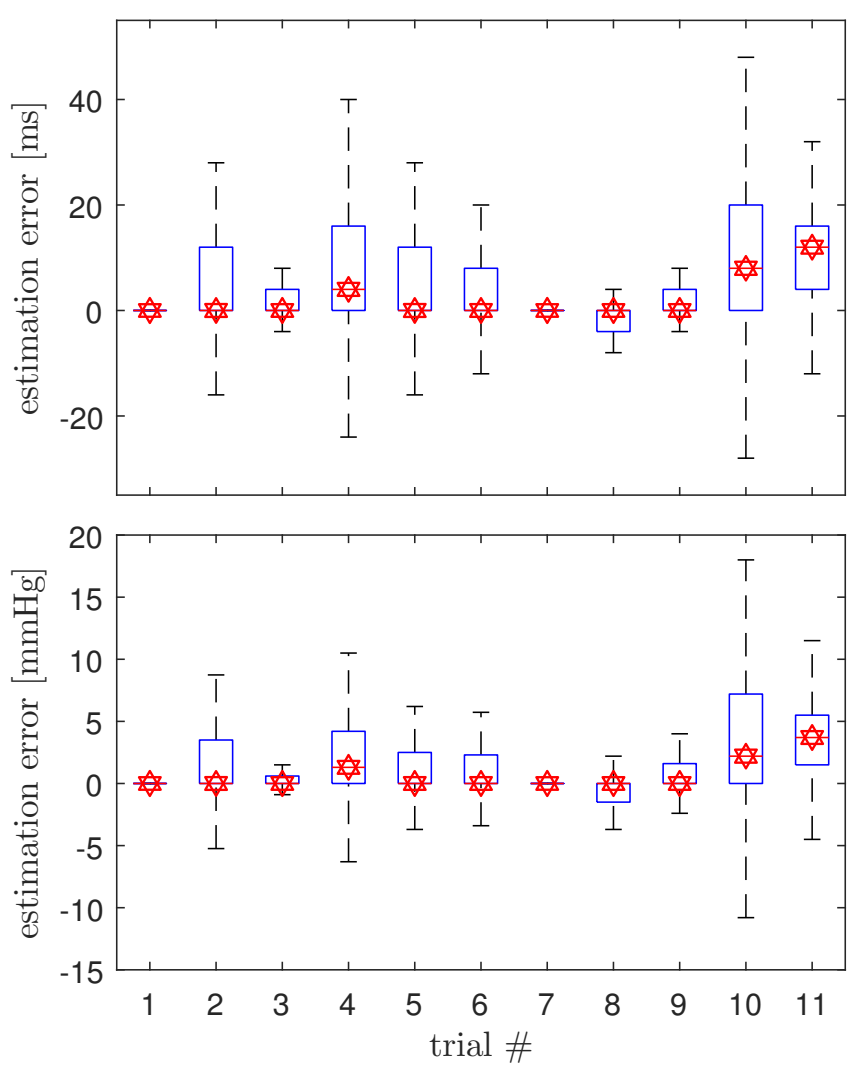

Figure 4. Boxplot of the cross-validated estimation error in terms of time and pressure value for $q=8$. Outliers are omitted for clarity reasons.

given in Table 2.

Several observations can be made. First, the estimation quality is rather homogeneous in terms of temporal accuracy. Here, a mean RMSE of $11.6 \mathrm{~ms}$ can be achieved when cross validation is applied. This error is slightly lower $(10.5 \mathrm{~ms})$, when no cross validation but the filters trained on the respective data are used. It is further worth noting that, for some cases, a comparatively large systematic error exists. Second, the estimation error in terms of the signal's amplitude differs strongly. While for some cases it lies in the range of the sensor accuracy, it comes close to the standard deviation of the EDP for others. Moreover, the difference between cross validation and us- 


\begin{tabular}{r|rrr|rrr} 
& \multicolumn{4}{|c|}{ estimation RMSE (mean error) } \\
trial & \multicolumn{3}{|c}{ [ms] } & \multicolumn{3}{c}{ [mmHg] } \\
\# & self & \multicolumn{2}{|c}{ cross-val. } & self & \multicolumn{2}{c}{ cross-val. } \\
\hline 1 & 8.6 & 10.0 & $(2.9)$ & 1.2 & 1.5 & $(0.4)$ \\
2 & 12.2 & 13.8 & $(6.4)$ & 3.6 & 4.5 & $(2.0)$ \\
3 & 7.6 & 8.3 & $(0.5)$ & 2.2 & 2.6 & $(0.0)$ \\
4 & 12.0 & 15.0 & $(9.2)$ & 3.0 & 4.1 & $(2.3)$ \\
5 & 12.9 & 13.2 & $(5.7)$ & 3.6 & 3.8 & $(0.9)$ \\
6 & 9.5 & 10.5 & $(3.0)$ & 3.1 & 3.5 & $(0.6)$ \\
7 & 3.7 & 3.7 & $(0.8)$ & 1.0 & 1.0 & $(0.2)$ \\
8 & 8.3 & 8.5 & $(-1.0)$ & 4.7 & 4.8 & $(-0.9)$ \\
9 & 11.2 & 10.9 & $(2.0)$ & 4.7 & 4.7 & $(0.0)$ \\
10 & 15.0 & 18.1 & $(11.4)$ & 4.5 & 6.3 & $(3.8)$ \\
11 & 14.6 & 16.0 & $(9.6)$ & 7.9 & 8.4 & $(1.6)$ \\
\hline$\varnothing$ & 10.5 & 11.6 & $(4.6)$ & 3.6 & 4.1 & $(1.0)$
\end{tabular}

Table 2. Temporal and pressure error in terms of RMSE as well as systematic offset for $q=8$. The last row shows the mean over all trials.

ing the signal-specific filter coefficients is more profound.

In [3], the difference between human annotation and the proposed peak curvature method was reported to be $-1.5(4.2) \mathrm{ms}$ in terms of mean(SD). For intra- and inter-observation error, these values were found to be $0.7(2.7) \mathrm{ms}$ and 2.6(3.4) ms, respectively. Thus, an acceptance region of $\pm 10.1 \mathrm{~ms}$ was defined. Considering these values, our method exhibits a comparable yet inferior performance. No results in terms of pressure accuracy were reported in [3].

While the exact reason for the varying performance of the proposed method need further investigation it should be noted that the performance using the optical sensor (type 'B') was notably higher than with the conventional sensor.

Animals from the range of "Fetus pre/post birth" to "2-3 year Sheep" were considered and a total of 2000 ED time points were annotated in [3]. In this trial, only adult sheep were considered. However, due to the provoked infarction an the subsequent administration of pharmaceuticals, severe changes in the sheep's CVS could be observed. In addition, a total of 201885 ED time points were manually annotated.

Finally, it should be noted that a basic peak selection algorithm was applied to the estimated signal $\tilde{y}(t)=$ $a(t) * x(t)$ only. Assuming a low computational cost for peak detection, this would allow the estimation of EDP with low latency for real-time applications, as filtering with $q=8$ introduces a delay of only $36 \mathrm{~ms}$. However, this strategy is likely suboptimal in terms of estimation error and could probably be improved by adding more features derived from the LVP signal.

\section{Conclusion}

In this paper, a novel method based on deconvolution of the LVP signal to estimate the ED point in time was proposed. The method was evaluated on a total of 37.6 hours of human annotated animal trial data. Using crossvalidation, a RMSE of $11.6 \mathrm{~ms}(4.1 \mathrm{mmHg})$ was achieved. This demonstrates comparability to methods proposed in the literature in [3]. It further shows the general applicability and the potential of deconvolution methods for feature extraction and processing of cardiac signals.

\section{References}

[1] Kirklin JK, Naftel DC, Pagani FD, Kormos RL, Stevenson LW, Blume ED, Myers SL, Miller MA, Baldwin JT, Young JB. Seventh intermacs annual report: 15,000 patients and counting. The Journal of Heart and Lung Transplantation 2015;34(12):1495-1504.

[2] AlOmari AHH, Savkin AV, Stevens M, Mason DG, Timms DL, Salamonsen RF, Lovell NH. Developments in control systems for rotary left ventricular assist devices for heart failure patients: a review. Physiological Measurement 2012; 34(1):R1.

[3] Mynard JP, Penny DJ, Smolich JJ. Accurate automatic detection of end-diastole from left ventricular pressure using peak curvature. IEEE Transactions on Biomedical Engineering 2008;55(11):2651-2657.

[4] Hoog Antink C, Brüser C, Leonhardt S. Multimodal sensor fusion of cardiac signals via blind deconvolution: a sourcefilter approach. In Computing in Cardiology Conference (CinC), 2014. IEEE, 2014; 805-808.

[5] Böhm A, Hoog Antink C, Leonhardt S, Teichmann D. Determining the connection between capacitively coupled electrocardiography data and the ground truth. In Computing in Cardiology Conference (CinC), 2015. IEEE, 2015; 677-680.

[6] He Z, Xie S, Fu Y. A new blind deconvolution algorithm for SIMO channel based on neural network. In Proceedings of the Fourth International Conference on Machine Learning and Cybernetics. 2005; 3602-3606.

[7] Abeysinghe DC, Dasgupta S, Boyd JT, Jackson HE. A novel MEMS pressure sensor fabricated on an optical fiber. IEEE Photonics Technology Letters 2001;13(9):993-995.

Address for correspondence:

Christoph Hoog Antink

Chair for Medical Information Technology

Helmholtz-Institut, RWTH Aachen

Pauwelsstr. 20 / D-52074 Aachen / Germany

hoog.antink@hia.rwth-aachen.de 